\title{
ESTUDO COMPARATIVO DO PREPARO TRANSVERSAL PRODUZIDO PELO EMPREGO DE INSTRUMENTOS DE NITI E MANUAL EM MOLARES INFERIORES
}

\author{
COMPARATIVE STUDY OF THE TRANSVERSAL PREPARATION CARRIED \\ OUT BY NITI AND MANUAL INSTRUMENTS IN LOWER MOLARS
}

\author{
Patrícia Correia de SIQUEIRA'; Gustavo Silva CHAVES'; Giulliano Caixeta SERPA'; Marco Antônio Zaiden LOUREIRO'; \\ Alessandro Moreira FREIRE'; Vinícius Caixeta de SOUSA ${ }^{1}$ \\ 1 - Faculdade de Odontologia da Universidade Federal de Goiás (UFG) \\ 2 - Faculdade de Odontologia da Faculdade Alfredo Nasser (UNIFAN-GO)
}

\section{RESUMO}

Objetivo: avaliar o desgaste dentinário promovido por instrumentação rotatória com diferentes sistemas de NiTi e instrumentação manual em raízes mesiais de molares inferiores. Material e Métodos: Foram selecionados 60 molares inferiores extraídos que foram divididos em 6 grupos $(n=10)$. Nos grupos A, B, C e D os canais radiculares foram preparados utilizando-se os sistemas de NiTi ProFile ${ }^{\circledR}$, ProTaper Universal ${ }^{\circledR}, \mathrm{K}^{\circledR} \mathrm{e} \mathrm{Race}^{\circledR}$, respectivamente. No grupo E, os canais radiculares foram preparados com limas manuais do tipo K Flexofile ${ }^{\circledR}$, e no grupo F os canais radiculares não foram preparados (controle). Após os preparos, foram realizados dois cortes perpendiculares ao longo eixo da raiz mesial, sendo um no terço cervical e outro no terço apical. As imagens dos cortes foram capturadas por meio de microscópio e analisadas através de software. Para cada corte, foram realizadas três medidas correspondentes à menor espessura da parede dentinária, sendo obtida uma média de cada espécime. As comparações entre os grupos das medidas obtidas foram realizadas pelo teste $t$, com nível de significância de 5\%. Resultados: Tanto no terço cervical quanto apical houve diferença significante entre o grupo E (Flexofile) e os grupos de sistemas de NiTi, bem como entre o grupo controle. No terço apical foi observada diferença significante entre o grupo A (Profile ${ }^{\circledR}$ ) e o grupo D (Race ${ }^{\circledR}$ ) em comparação ao grupo controle. Conclusões: Pôde-se concluir que as técnicas que utilizaram instrumentos de NiTi preservaram mais estrutura dentinária quando comparados à técnica que se utilizou de limas manuais.

Palavras-chave: endodontia, dente molar, desgaste dentário.

\section{INTRODUCÃO}

O tratamento endodôntico é constituído por várias etapas, que incluem adequada abertura coronária, correto preparo do canal radicular, utilização de medicação intracanal quando há presença de infecção, selamento do sistema de canais radiculares por meio de uma obturação satisfatória, assim como um bom selamento coronário e proservação ${ }^{1}$.

Durante o preparo do canal radicular, alguns aspectos devem ser observados para se obter o êxito no tratamento, como a escolha do instrumento ideal e o respeito aos princípios biológicos e mecânicos. Dessa forma, o instrumento de escolha para a realização do preparo do canal radicular deve ser capaz de fornecer um preparo que não fuja desses princípios, ou seja, capaz de manter a forma original do canal, sem promover um desgaste excessivo das paredes dentinárias ${ }^{1}$.

Dessa forma, foram desenvolvidos os instrumentos de Níquel-Titânio (NiTi), que apresentam alta flexibilidade e memória elástica. A liga de níquel-titânio (NiTi) foi desenvolvida no Naval Ordenance Laboratory (NOL) nos Estados Unidos, razão pela qual recebeu o nome de NiTiNOL ${ }^{2}$. Esses instrumentos revolucionaram o tratamento endodôntico reduzindo a fatiga do operador e o tempo necessário para a conclusão do preparo do canal radicular, associado à diminuição de desvio dos canais durante a instrumentação ${ }^{3}$. São instrumentos fabricados por usinagem, a partir de uma haste cônica metálica de secção circular, com $55-60 \%$ de níquel, $40-45 \%$ de titânio, baixo módulo de elasticidade, efeito memória de forma e superelasticidade ${ }^{4}$. Desde a introdução destes instrumentos na endodontia, uma grande variedade de sistemas tem sido lançada, cada um com características específicas.

Estrela \& Stephan ${ }^{5}$ (1991) salientam o cuidado que deve ser tomado durante o preparo do terço cervical de raízes mesiais de molares inferiores. A raiz mesial do $1^{0}$ molar inferior possui uma concavidade sobre a superfície distal. O canal mésio-vestibular tem seu maior diâmetro no sentido mésio-distal, com aspecto de "vírgula". Frequentemente acontece de os canais mésio-vestibular e mésio-lingual estarem mais próximos da superfície distal do que aparece na radiografia, e dessa forma, especial atenção deve ser tomada durante o preparo dessa região.

Outro aspecto observado no preparo dos canais curvos, seja por meio de instrumentos manuais ou acionados a motor elétrico, é o desvio do canal na região apical e a formação de "zips", levando a um maior desgaste nas paredes dentinárias da região apical ${ }^{6}$. 
Esse desgaste excessivo das paredes dentinárias, tanto em nível cervical quanto apical, pode levar a formação de paredes finas ou mesmo perfurações nessas regiões, conduzindo ao insucesso do tratamento. Dessa forma, torna-se fundamental avaliar o desgaste das paredes dentinárias em diferente níveis da raiz produzido por técnicas rotatórias de instrumentação dos canais radiculares com instrumentos de $\mathrm{NiTi}$, comparando-as à instrumentação manual, realizada com instrumentos de aço inoxidável.

O objetivo do presente estudo foi avaliar o desgaste dentinário promovido por diferentes sistemas rotatórios com instrumentos de NiTi (ProFile ${ }^{\circledR}$, Protaper Universal ${ }^{\circledR}, \mathrm{K}^{\circledR}{ }^{\circledR}$ e Race ${ }^{\circledR}$ ) e manual (K-Flexofile ${ }^{\circledR}$ ), em raízes mesiais de primeiros molares inferiores, nos terços cervical e apical.

\section{MATERIAL E MÉTODOS}

O modelo experimental utilizado neste estudo foi inicialmente modificado de Estrela \& Stephan ${ }^{5}$ (1991).

\section{Seleção e distribuição das amostras}

Para o presente estudo foram selecionados 60 primeiros molares inferiores humanos extraídos permanentes. Os critérios de inclusão foram dentes com rizogênese completa, presença de três canais radiculares e raiz mesial com curvatura moderada (aproximadamente $25^{\circ}$ ). Foram excluídos dentes com calcificações pulpares, obliteração do canal radicular, reabsorções radiculares e tratamento endodôntico prévio.

A abertura coronária foi realizada em todos os dentes com pontas diamantadas 1013 (KG Soresen, SP, Brasil) em alta rotação sob constante refrigeração, e em seguida os canais radiculares foram explorados com um instrumento do tipo K\#10 (Dentsply, Tulsa, USA) e esvaziados até um instrumento K Flexofile ${ }^{\circledR}$ \#15 (Dentsply, Tulsa, USA). A odontometria foi obtida com o instrumento inicial introduzido no canal até alcançar o forame apical. A partir daí, por visão direta do ápice, recuava-se a lima $1 \mathrm{~mm}$ para determinação do comprimento de trabalho.

Os dentes foram distribuídos de forma aleatória em 6 grupos $(n=10)$, de acordo com o sistema utilizado para o preparo dos canais radiculares mesiais de cada dente (Quadro 1). No grupo A, utilizou-se o sistema ProFile ${ }^{\circledR}$ (Dentsply, Tulsa, USA); no grupo B o sistema ProTaper Universal ${ }^{\circledR}$ (Dentsply, Tulsa, USA); no grupo $\mathrm{C}$ o sistema $\mathrm{K}^{\circledR}{ }^{\circledR}$ (SybronEndo, Orange, CA); no Grupo D o sistema $\mathrm{RaCe}^{\circledast}$ (FKG, La Chaux-de-Fonds, Suíça) e no Grupo E instrumentos manuais de aço inoxidável K Flexofile ${ }^{\circledR}$ (Dentsply, Tulsa, USA). O grupo F foi o controle, no qual os dentes não foram submetidos a nenhum tipo de preparo do canal radicular.

\section{Preparo dos canais radiculares}

Para a instrumentação dos canais radiculares, os dentes foram presos pela coroa a uma prensa fixada à mesa de trabalho, procurando obedecer à correta cinemática dos instrumentos preconizada pelas distintas técnicas, sem interferências. Em todos os grupos foram preparados os dois canais mesiais (mésio-vestibular e mésio-lingual) e a solução irrigadora utilizada foi o hipoclorito de sódio a 1\% (Halexstar ${ }^{\circledR}$, Goiânia, Brasil), com volume de $3 \mathrm{~mL}$ de solução após a troca de cada lima.

Para a realização dos preparos automatizados foi utilizado o
Quadro 1 - Distribuição dos grupos experimentais.

\begin{tabular}{|c|c|c|}
\hline GRUPO & TÉCNICA DE INSTRUMENTAÇÃO & $\mathrm{n}$ \\
\hline$A$ & $\begin{array}{l}\text { Sistema ProFile } \\
15.02 \text { / } 20.02 \text { / } 25.02 \text { / } 30.02 \text { / } 35.02\end{array}$ & 10 \\
\hline B & $\begin{array}{l}\text { Sistema ProTaper universal }{ }^{\circledR} \\
\text { SX (19.04) / S1 (18.02) / S2 (20.04) / F1 (20.07) / F2 (25.08) / F3 } \\
\text { (30.09) }\end{array}$ & 10 \\
\hline$C$ & $\begin{array}{l}\text { Sistema K3 }{ }^{\circledR} \\
15.02 / 20.02 / 25.02 / 30.02 / 35.02\end{array}$ & 10 \\
\hline$D$ & $\begin{array}{l}\text { Sistema Race }{ }^{\circledR} \\
\text { Pré-RaCe }(40.10 \text { / 35.08) / } 25.06 / 25.04 / 25.02\end{array}$ & 10 \\
\hline$E$ & $\begin{array}{l}\text { Instrumentos manuais - K Flexofile }{ }^{\circledR} \\
15.02 \text { / } 20.02 \text { / } 25.02 \text { / } 30.02 \text { / } 35.02\end{array}$ & 10 \\
\hline $\mathrm{F}$ & Controle - sem preparo & 10 \\
\hline
\end{tabular}

motor elétrico TC Motor 3000 (Nouvag, Goldach, Switzerland), ajustado com velocidade de $300 \mathrm{rpm}$ e torque $4 \mathrm{~N} / \mathrm{cm}$. O preparo dos canais radiculares com instrumentos de NiTi foi realizado utilizando cinemática de acordo com as instruções do fabricante, usando um leve movimento de entrada e saída com avanços em direção apical de forma lenta e amplitude de aproximadamente $0,5 \mathrm{~mm}$.

Para o grupo E realizou-se o preparo do terço cervical com brocas de Gates Glidden nos 1 e 2 (Dentsply, Tulsa, USA) na profundidade de 15 a $17 \mathrm{~mm}$ e o posteriormente o preparo do terço apical com limas K Flexofile ${ }^{\circledR}$.

No grupo F, dez dentes foram completamente esvaziados com uma lima K-FlexoFile ${ }^{\circledR}$ de $n^{\circ} 15$ e não foram preparados, servindo como controle.

\section{Preparo das amostras e análise histomorfométrica}

Após o preparo dos canais radiculares, foram realizados dois cortes perpendiculares ao longo eixo da raiz mesial, utilizando-se um disco diamantado ultrafino $7 / 8\left(\right.$ Adaco $^{\circledR}$ ) com corte em apenas um lado, montado num micromotor. $\mathrm{O}$ primeiro corte foi feito a $2 \mathrm{~mm}$ abaixo da bifurcação radicular e o segundo a 2 $\mathrm{mm}$ aquém do ápice radicular visível.

Em seguida, os cortes passaram por um processo de desgaste, utilizando-se uma lixa d'água de granulação 220 (Norton ${ }^{\circledR}$ ), e por um processo de limpeza, com um banho de EDTA trissódico à $17 \%$ por um período de $3 \mathrm{mim}$, para remoção dos debris dentinários, provenientes das fases de preparo e obtenção dos cortes.

As imagens dos cortes foram capturadas por meio de microscópio de luz (Olympus ${ }^{\circledR}$ Triocular BH2), acoplado a uma câmera de vídeo (JVC ${ }^{\circledR} \mathrm{JK}-1085 \mathrm{U}$ ) ligada a um computador e analisadas através do software HLImage 97 (Western Vision ${ }^{\circledR}$ Softwares). Em cada corte, foram realizadas três medidas da menor espessura da parede dentinária, e uma média de cada espécime foi obtida (Figura 1). As medidas foram realizadas em micrômetros $(\mu \mathrm{m})$.

\section{Análise estatística}

As medidas foram tabuladas e analisadas pelo programa BioEstat versão 5.3. Inicialmente os dados foram analisados quanto à distribuição pelo teste Kolmogorov-Smirnov. Como foi observada distribuição paramétrica, utilizou-se o test $\mathrm{t}$ para as comparações entre os grupos. O nível de significância adotado foi de $5 \%(\alpha=0,05)$. 


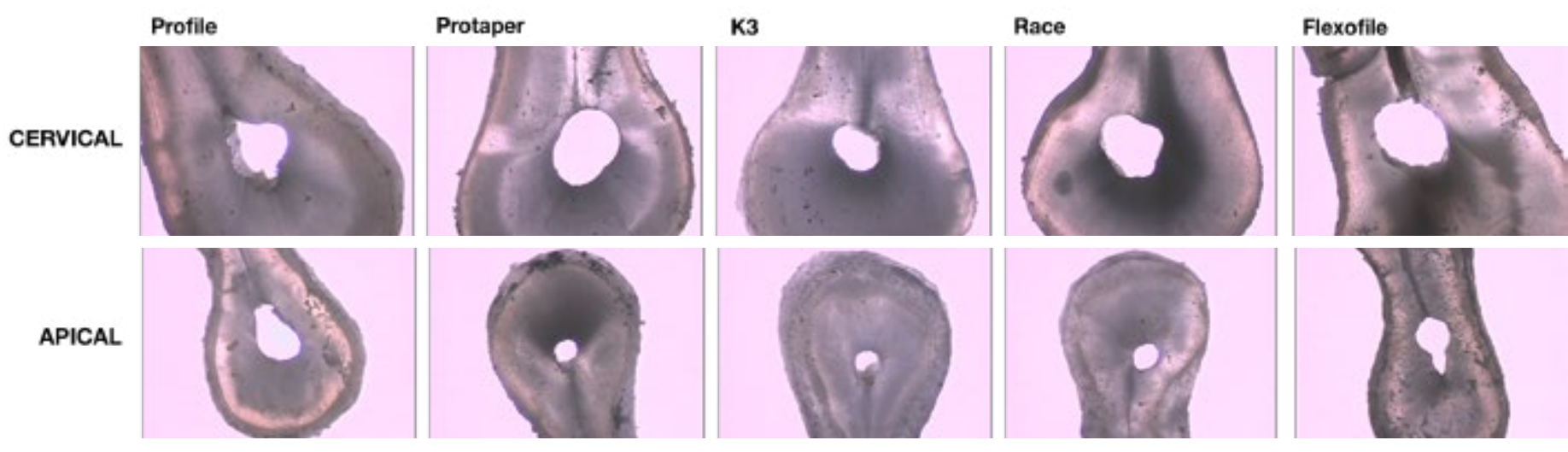

Figura 2 - Imagens representativas dos cortes de cada grupo experimental registradas no microscópio de luz.

\section{RESULTADOS}

A Figura 2 apresenta imagens representativas de cada grupo experimental tanto no terço cervical quanto no terço apical, registradas através do microscópio de luz.

As médias de espessura dentinária obtidas nos cortes tanto na região cervical quanto apical em cada grupo estão descritas na Tabela 1. Tanto no terço cervical quanto no terço apical, observou-se diferença significante entre o grupo E (K-Flexofile ${ }^{\circledR}$ ) em comparação aos grupos de sistemas de NiTi e ao grupo controle $(p<0,05)$. No terço apical também foi observada diferença estatisticamente significante entre o grupo A (Profile ${ }^{\circledR}$ ) e o grupo D $\left(\right.$ Race $\left.^{\circledR}\right)$ em comparação ao grupo controle $(p<0,05)$.

A Figura 3 e 4 apresentam os gráficos representativos da distribuição dos valores obtidos em cada grupo no terço cervical e apical, respectivamente.

\section{DISCUSSÃO}

O sucesso do tratamento endodôntico está fundamentado em um adequado processo de sanificação dos canais radiculares infectados. Para isso, é fundamental um esvaziamento e alargamento do canal radicular, bem planejados e executados, que resulte em um maior controle microbiano ${ }^{1}$.

O objetivo do presente experimento foi avaliar o desgaste dentinário promovido por técnicas de instrumentação rotatórias realizadas com instrumentos de NiTi e manuais, em raízes mesiais de primeiros molares inferiores (terços cervical e apical). $\mathrm{O}$ modelo de estudo adotado foi adaptado de prévia investigação que comparou técnicas de instrumentação manual ${ }^{5}$, analisando-se o desgaste dentinário em nível cervical, região correspondente à zona crítica de perfuração ${ }^{7}$.

Um dos questionamentos diz respeito ao limite de alargamento transversal, que não apresente risco de perfuração ou desgaste exagerado em áreas críticas do canal radicular. Os resultados mostraram que as técnicas de instrumentação proporcionaram desgastes dentinários distintos. O emprego de instrumentos de $\mathrm{NiTi}$, independente do sistema, preservou estrutura dentinária tanto em nível cervical quanto apical, com valores significantes quando comparados à técnica em que se utilizou de limas manuais.

Diversos estudos foram realizados acerca da avaliação do desgaste dentinário promovido pelo preparo do canal radicular. Estrela \& Stephan ${ }^{5}$ (1991) estudaram comparativamente o desgaste dentinário na parede distal do canal mésio-vestibular do primeiro molar inferior, produzido por três técnicas de instrumentação.

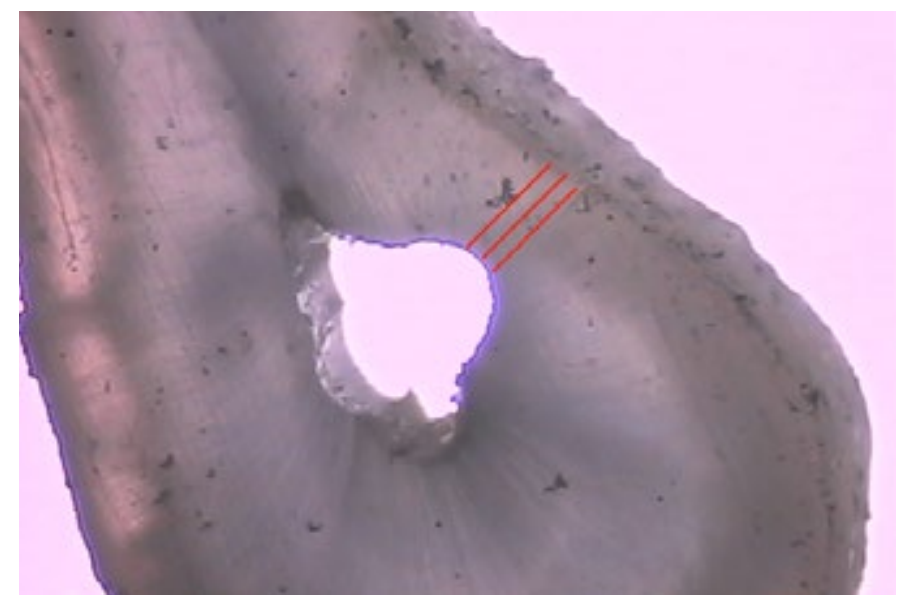

Figura 1 - Imagem de um corte observado no microscópio ilustrando os locais nos quais foram realizadas as medidas (linhas em vermelho), na região de menor espessura de dentina.

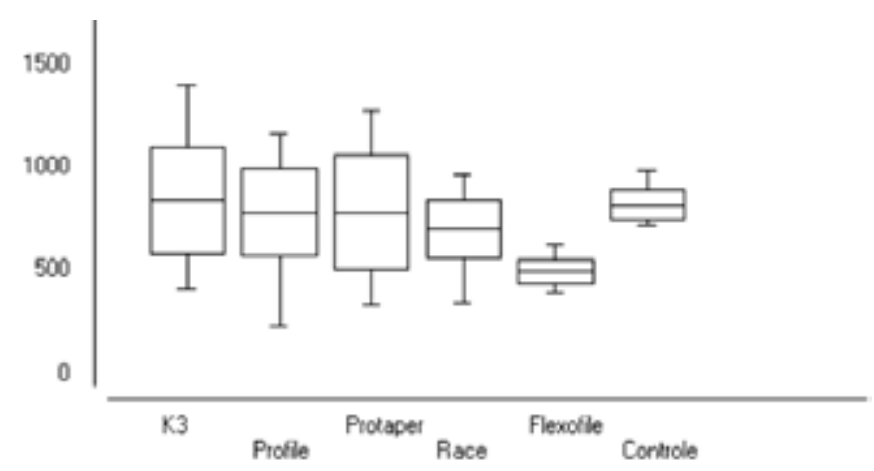

Figura 3 - Gráfico representando a distribuição dos valores encontrados em cada grupo no terço cervical.

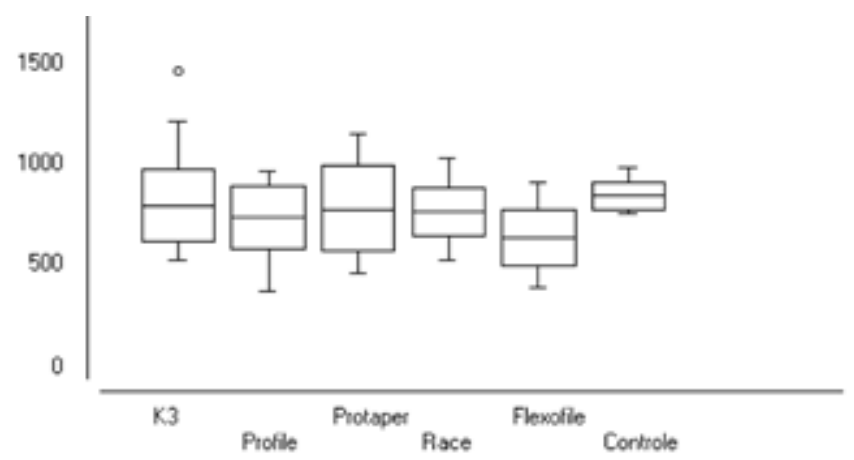

Figura 4 - Gráfico representando a distribuição dos valores encontrados em cada grupo no terço apical. 
Os canais radiculares de 80 dentes foram preparados por três técnicas diferentes: anticurvatura, telescópica e convencional. Terminado o preparo dos canais, foram feitos, quatro cortes perpendiculares ao longo eixo da raiz mesial. Os autores observaram que a instrumentação pela técnica da anticurvatura proporciona maior segurança, sendo a de menor risco de perfuração e de criação de paredes finas, sendo que esta foi a única técnica que deixou uma espessura média maior que $0,5 \mathrm{~mm}$ de dentina na parede distal. Os canais instrumentados pela técnica convencional auxiliada pelas brocas Gates-Glidden foram os que ficaram com paredes mais finas, apresentando os mais baixos índices de espessura. Esta técnica apresenta maior possibilidade de perfuração. Outro ponto observado pelos autores foi que nas três técnicas de instrumentação, a região próxima à bifurcação foi o local de maior susceptibilidade da ocorrência de perfuração.

Estrela et al. ${ }^{8}$ (1993) estudaram a formação de "zip" apical, a partir da variação do instrumento memória em canais radiculares curvos. Utilizou-de 75 canais simulados, confeccionados em blocos de resina epóxi, com curvaturas de 40 graus. Determinado o comprimento de trabalho, recobriram-se com fita isolante os blocos de resina, garantindo que os preparos fossem feitos sem influências ditadas pela visualização. Os canais foram preparados utilizando-se limas K-Flexofile ${ }^{\circledR}$, pré-curvadas em 40 graus. Os canais foram divididos em três grupos, onde se desenvolveu a técnica escalonada, variando-se o instrumento memória nos números 25, 30 e 35. Uma vez preparados, todos os blocos foram avaliados visualmente quanto á presença ou à ausência do "zip" apical. O grupo 1, cujo instrumento memória era o de número 25 , obteve a menor incidência de formação de "zip" apical, numa porcentagem de $32 \%$. Os de número 30 exibiram um índice maior, 56\% de "zip" apical, num total de 14 blocos. No grupo 3, que teve como memória o instrumento de número 35, observou-se a maior incidência de "zip" apical, 76\%, em 19 blocos.

Na busca por instrumentos que proporcionassem menor desvio do canal radicular e consequente menor desgaste, foi introduzida a liga de níquel-titânio (NiTi). Walia et al. ${ }^{2}$ (1988) realizaram uma investigação inicial do uso das ligas de NiTi em endodontia com a utilização de limas \#15 produzidas experimentalmente a partir de fios ortodônticos compostos por estas ligas. Limas endodônticas de secção triangular foram fabricadas através de um arco de NiTinol de 0,02 polegadas, uma liga ortodôntica de níquel-titânio com um módulo de elasticidade muito baixo. As limas de NiTinol apresentaram de 2 a 3 vezes mais flexibilidade elástica na torsão e dobramento, assim como superior resistência à fratura torsional, quando comparada a uma lima de \#15 de aço inoxidável fabricada pelo mesmo processo. As superfícies das fraturas torsionais no sentido horário e anti-horário foram analisadas por um microscópio eletrônico de varredura e mostraram morfologias planas para as limas dos dois tipos de liga e os dois modos de teste torsional. Os resultados deste estudo sugeriram a possibilidade de uma nova geração de limas, possuindo um grau de flexibilidade que pode ser ideal para instrumentação de canais curvos.

Assim, buscando comparar os instrumentos de NiTi com instrumentos manuais, vários estudos foram realizados. Esposito \& Cunningham ${ }^{9}$ (1995) compararam a formatação de canais radiculares curvos durante o preparo com limas manuais de níquel-titânio $\mathrm{Mac}^{\circledR}$, limas de níquel-titânio $\mathrm{NT}^{\circledR}$ acionadas por motor e limas de aço inoxidável K-Flex ${ }^{\circledR}$ em quarenta e cinco dentes humanos extraídos. As limas de níquel-titânio foram mais efetivas na manutenção do trajeto original de canais radiculares curvos quando o preparo apical foi dilatado além do tamanho $n^{\circ}$ 30. Os resultados mostraram que os instrumentos de NiTi foram mais efetivos do que as limas de aço inoxidável na manutenção do trajeto original dos canais radiculares curvos quando o preparo apical foi alargado ao tamanho $n^{\circ} 35, n^{\circ} 40$ ou $n^{\circ} 45$. Os instrumentos de NiTi permitiram preparos apicais dos canais radiculares mais amplos, mantendo o trajeto original do canal.

Sendo assim, diversas configurações e marcas de instrumentos fabricados a partir da liga de níquel-titânio foram desenvolvidas, buscando exatamente diminuir alterações estruturais no preparo do canal radicular e risco de fratura dos instrumentos. Iqbal et al. ${ }^{3}$ (2004) usaram uma técnica radiográfica para comparar o transporte apical e a perda do comprimento de trabalho entre instrumentos rotatórios de NiTi ProFile ${ }^{\circledR}$ e ProTaper ${ }^{\circledR}$. Foram utilizados canais mésio-vestibulares de 40 molares inferiores extraídos. Os resultados indicaram que tanto ambos os sistemas foram similares na habilidade de promover alargamento do canal radicular com o mínimo de transporte e perda do comprimento de trabalho.

Schäfer \& Vlassis ${ }^{10}$ (2004) compararam os instrumentos do sistema ProTaper ${ }^{\circledast}$ com o sistema RaCe ${ }^{\circledR}$ durante a modelagem de canais simulados em blocos de resina, com grau de curvatura de $28^{\circ}$ ou $35^{\circ}$. Os canais simulados foram preparados com um dos sistemas avaliados por um operador experiente. De acordo com os autores, os resultados dessa investigação confirmam os resultados de estudos anteriores com diferentes instrumentos rotatórios de níquel-titânio, salientando sua habilidade de manter a forma original de canais extremamente curvos. Dentro das limitações do presente estudo, os instrumentos ProTaper ${ }^{\circledR}$ e $\mathrm{RaCe}^{\circledR}$ preparam canais curvos rapidamente e foram relativamente seguros. Em ambos os tipos de canal, os instrumentos $\mathrm{RaCe}^{\circledast}$ mantiveram a curvatura original do canal melhor que o ProTaper $^{\circledR}$, os quais tenderam o transporte em direção à posição mais externa da curvatura na região mais apical dos canais.

Em outro estudo, Schäfer \& Vlassis ${ }^{11}$ (2004) compararam a efetividade de limpeza após o preparo de canais radiculares com curvaturas severas com os instrumentos ProTaper ${ }^{\circledR}$ e RaCe ${ }^{\circledR}$, além de avaliar se a instrumentação tinha efeito na curvatura do canal radicular em dentes humanos extraídos. Com ambos os instrumentos, nenhum canal apresentou sobre-extensão do preparo, enquanto que a perda do comprimento de trabalho foi encontrada em quatro canais preparados com ProTaper ${ }^{\circledR}$ e três canais alargados com $\mathrm{RaCe}^{\circledR}$. Em relação à média de regularização dos canais curvos, o uso dos instrumentos $\mathrm{RaCe}^{\circledR}$ resultou significantemente em menor regularização durante a instrumentação comparado com os instrumentos ProTaper ${ }^{\circledR}$. Acerca dos parâmetros desse estudo, o uso das limas $\mathrm{RaCe}^{\circledR}$ resultou em redução significativamente menor de debris comparado com o preparo do instrumento ProTaper $^{\circledR}$, enquanto que em ternos de smear layer, não foi visível nenhuma diferença estatisticamente significante. As limas $\mathrm{RaCe}^{\circledR}$ mantiveram a curvatura original significantemente melhor que o ProTaper $^{\circledR}$.

Calberson et al. ${ }^{12}$ (2004) investigaram a capacidade de modelagem das limas ProTaper ${ }^{\circledR}$ em canais radiculares simulados curvos. Para isso, mediram a quantidade de material removido nos diferentes níveis do canal radicular e registraram as aberrações 
que foram criadas após o preparo. Os canais simulados foram divididos em quatro grupos diferentes, sendo as variações quanto ao ângulo de curvatura (um $20^{\circ}$ e o outro $40^{\circ}$ ) e à posição da curvatura (porção reta antes da curva com 8 ou $12 \mathrm{~mm}$ de comprimento). Os resultados dos preparos dos canais foram avaliados por uma câmera digital conectada a um computador. As imagens pré e pós-operatórias foram armazenadas e editadas no programa Photoshop 6 e as mensurações foram realizadas usando um programa para análise de imagens. Os autores relataram que em canais estreitos e curvos, o comprimento da porção reta do canal determinou a direção de transporte mais que o ângulo de curvatura. Sem levar em consideração o tipo de canal usado no estudo, os instrumentos ProTaper ${ }^{\circledR}$ removeram mais resina do lado interno da curvatura em comparação ao lado externo da curvatura. Sob as condições do estudo, os instrumentos ProTaper $^{\circledR}$ produziram aberrações após o uso dos instrumentos F2 e F3. Atenção deve ser tomada com o uso desses instrumentos para evitar a remoção excessiva na parte interna da curvatura, em direção à zona de perigo, e a retificação do canal radicular. Quando utilizar F3 em canais curvos, tomar cuidados também para evitar a deformação do instrumento.

Paqué et al. ${ }^{13}$ (2005) compararam vários parâmetros de preparo do canal radicular utilizando os instrumentos rotatórios de $\mathrm{NiTi}$ $\mathrm{RaCe}^{\circledR} \mathrm{e}$ ProTaper ${ }^{\circledR}$. Foram avaliados molares inferiores extraídos com canais radiculares mesiais de curvatura entre $20^{\circ}$ e $40^{\circ}$. Os canais radiculares foram preparados por instrumentos rotatórios $\mathrm{RaCe}^{\circledR}$ ou ProTaper ${ }^{\circledR}$ e os seguintes parâmetros foram avaliados: retificação de canais curvos, secção transversal do canal radicular pós-preparo, questões de segurança e tempo de trabalho. Ambos os sistemas de NiTi mantiveram bem a curvatura original. No preparo com a utilização dos instrumentos $\mathrm{RaCe}^{\circledR}, 49 \%$ dos canais radiculares apresentaram diâmetro arredondados ou ovalados e $50 \%$ de diâmetros irregulares, enquanto o sistema ProTaper ${ }^{\circledR}$ resultou em diâmetros arredondados ou ovalados em $50 \%$ dos casos. Houve perda de comprimento de trabalho em duas raízes com os instrumentos $\mathrm{RaCe}^{\circledR}$, enquanto que o preparo com ProTaper ${ }^{\circledR}$ resultou em perda de comprimento de trabalho em duas raízes e uma fratura de instrumento. O tempo de trabalho foi menor para ProTaper ${ }^{\circledR}$ do que para o $\mathrm{RaCe}^{\circledR}$ e ambos os sistemas respeitaram a curvatura original do canal radicular e foram seguros para o uso, porém a limpeza não foi satisfatória em ambos os sistemas.

\section{CONCLUSÕES}

Baseado na metodologia apresentada pode-se concluir que as técnicas que utilizaram instrumentos de Níquel-Titânio preservaram mais estrutura dentinária, tanto em nível cervical quanto apical quando comparados à técnica que se utilizou de limas manuais.

\section{REFERÊNCIAS BIBLIOGRÁFICAS}

01. Estrela C. Ciência Endodôntica. São Paulo: Artes Médicas, 2004. 1010 p.

02. Walia H, Brantley WA, Gerstein H. An initial investigation of the bending and torsional properties of nitinol root canal files. J Endod 1988; 14: 346-51.

03. Iqbal MK, Firic S, Tulcan J, Karabucak B, Kim S. Comparison of apical transportation between ProFile and ProTaper NiTi rotary instruments. Int Endod J 2004; 37: 359-64.

04. Serene TP, Adams JD, Saxena A. Nickel-Titanium instruments: applications in Endodontics. Ihiyama Euro America Inc: St Louis; 1995. 112p.

05. Estrela C, Stephan IW. Estudo comparativo do desgaste dentinário na parede distal do canal mésio-vestibular do primeiro molar inferior, produzido por três técnicas de instrumentação. Rev Odontol Brasil Central 1991, 1: 11-5.

06. Garcia FLB, Lopes HP, Lithgow CV. Avaliação do deslocamento do preparo do canal após o emprego de instrumentos endodônticos de aço inoxidável e de níquel-titânio da série 29. JBE - Jornal Brasileiro de endo/perio 2000; 3: 27-31.

07. Abou-Rass M, Frank AL, Glick DH. The anticurvature filling method to prepare the curve root canal. J Amer Dent Ass 1980; 101: 792-4.

08. Estrela C, Figueiredo JAP, Pesce HF. Avaliação da ocorrência de desvio apical, tendo como fonte de variação o instrumento memória quando do emprego da técnica escalonada. Rev Bras Odontol 1993. 50: 3-6.

09. Esposito PT, Cunningham CJ. A comparison of the canal preparation with nickel-titanium and stainless steel instruments. J Endod 1995; 21:173-6.

10. Schäfer E, Vlassis M. Comparative investigation of two rotary nickeltitanium instruments: ProTaper versus RaCe. Part 1. Shaping ability in simulated curved canals. Int Endod J 2004; 37: 229-38a.

11. Schäfer E, Vlassis M. Comparative investigation of two rotary nickel-titanium instruments: ProTaper versus RaCe. Part 2. Cleaning effectiveness and shaping ability in severely curved root canals of extracted teeth. Int Endod J 2004; 37: 239-48b.

12. Calberson FLG, Deroose CAJG, Hommez GMG, De Moor RJG. Shaping ability of ProTaper nickel-titanium files in simulated resin root canals. Int Endod J 2004, 37; 613-23.

13. Paqué F, Musch U, Hülsmann M. Comparison of root canal preparation using $\mathrm{RaCe}$ and ProTaper rotary $\mathrm{Ni}-\mathrm{Ti}$ instruments. Int Endod J 2005; 38: 08-16.

\section{ABSTRACT}

Objective: to evaluate dentinal wear promoted by rotary instrumentation with different NiTi systems and manual instrumentation in mesial roots of lower molars. Material and Methods: Sixty extracted lower molars were selected and divided into 6 groups $(n=10)$. In groups $A, B, C$ and $D$ the root canals were prepared using the NiTi ProFile ${ }^{\circledR}$, ProTaper Universal ${ }^{\circledR}, \mathrm{K}^{\circledR}$ and Race $^{\circledR}$ systems, respectively. In group E, the root canals were prepared with manual files of type $\mathrm{K}$ Flexofile ${ }^{\circledast}$, and in group $\mathrm{F}$ the root canals were not prepared (control). After the preparation, two perpendicular cuts were made along the axis of the mesial root, one in the cervical third and the other in the apical third. The images of the cuts were captured by means of a microscope and analyzed through software. For each cut, three measurements were made corresponding to the lowest thickness of the dentin wall, obtaining an average of each specimen. The comparisons between the groups of measures obtained were 
performed by the $t$ test, with a significance level of $5 \%$. Results: In both the cervical and apical thirds there was a significant difference between the group $\mathrm{E}$ (Flexofile) and the groups of $\mathrm{NiTi}$ systems, as well as between the control group. In the apical third, a significant difference was observed between group A (Profile ${ }^{\circledR}$ ) and group D (Race ${ }^{\circledR}$ ) compared to the control group. Conclusions: It could be concluded that the techniques that used NiTi instruments preserved more dentin structure when compared to the technique used of manual files.

Keywords: endodontics, molar, tooth wear

\section{AUTOR PARA CORRESPONDÊNCIA}

Profa. Dra. Patricia Correia de Siqueira

Faculdade de Odontologia / Universidade Federal de Goiás

Praça Universitária s/n, Setor Universitário

CEP: 74605-220, Goiânia, GO, Brazil.

Telefone: +55 (62) 3209-6254

e-mail: patricia.csiqueira@hotmail.com 